\title{
Die Privatisierung der Rentenversicherung in Chile
}

\author{
von Maximilian Fuchs
}

Die Reform der Rentenversicherung in Chile hat weltweit Schlagzeilen gemacht. Ihre Einmaligkeit besteht darin, daß zum ersten Mal in der Geschichte der Sozialversicherung ein Land mit einer langen Tradition klassischer Sozialversicherung sein öffentliches Rentenversicherungssystem aufgegeben und durch ein Privatversicherungsmodell abgelöst hat.

\section{Die Wirtschafts- und Sozialentwicklung Chiles seit dem Jahre 1973}

Die Wirtschafts- und Sozialentwicklung seit dem Sturz Allendes und der Ubernahme der Macht durch das Militärregime ist ausschließlich von der neoliberalen Wirtschaftstheorie der Friedmanschen Schule bestimmt gewesen. ${ }^{1}$ Die Zielrichtung der neuen Machthaber war es, das von vorangegangenen Regierungen betriebene Entwicklungsmodell der Importsubstitutionspolitik durch eine Exportorientierung der Wirtschaft abzulösen. Den Kritikern zufolge habe die Importsubstitutionspolitik zu einer Mißachtung der Marktgesetze, insbesondere der Wettbewerbsgrundsätze, geführt und durch eine protektionistische Politik Industrien gefördert, die nach der Theorie der komparativen Kosten nur durch die künstliche Unterstützung des Staates überlebensfähig waren, so daß das Bruttosozialprodukt nur sehr langsam gewachsen sei. ${ }^{2}$

Die Lösung der wirtschaftlichen Probleme konnte der neoliberalen Wirtschaftsauffassung zufolge nur durch folgende Strategien erreicht werden:

1. Es galt, einen dynamischen Kapitalmarkt zu schaffen, der die private Spartätigkeit anregt und Investitionen in produktiven Bereichen fördert.

2. Die protektionistische Politik mit ihren Zollschranken und Restriktionen mußte aufgegeben werden, um einen wirklichen Wettbewerb zu fördern und damit die finanzielle Misere, insbesondere das hohe Zahlungsbilanzdefizit abzubauen.

3. Die Staatstätigkeit mußte insgesamt eingeschränkt und durch eine umfassende Privatisierung aller wesentlichen Bereiche der Gesellschaft ersetzt werden. ${ }^{3}$

1 Die Beratertätigkeit Friedmans und Harbergers hat international heftige Kritik ausgelöst, vgl. den offenen Briefwechsel zwischen Frank, Friedman und Tintner, in: Neues Forum, 1976, Heft Juli/August, S. 23-34.

2 Dazu Rivera, E., Die chilenische Wirtschaftspolitik und die Umwandlung der Okonomie unter der Militärregierung (1973-1980), in: Calderón, H./Ensignia, J./Rivera, E., Chile. Der Monetarismus an der Macht, Hamburg, 1981, S. 61.

3 Rivera, E. (Fn. 2), S. $63 \mathrm{f}$. 
Es besteht kein Zweifel, daß die Verfolgung dieser wirtschaftlichen Maßnahmen nicht ohne Erfolg geblieben ist. Die Inflationsrate konnte auf ein erträgliches Maß reduziert und das Wirtschaftswachstum entscheidend gesteigert werden. ${ }^{4}$ Gleichwohl ist nicht zu verkennen, daß diese Erfolge, die im übrigen mit Rigidität und Repression durchgesetzt wurden, nur einer Minderheit zugute kamen und insbesondere große Teile der Lohnbevölkerung, aber auch die Mittelschichten enorme Einbußen hinnehmen mußten. ${ }^{5}$ Bei den Lohnerhöhungen der letzten Jahre wurden die höheren Schichten überdurchschnittlich bevorzugt. ${ }^{6}$ Man darf zudem nicht übersehen, daß erst 1978 (wahrscheinlich sogar erst 1979) wieder das Reallohnniveau von 1970 erreicht worden ist. ${ }^{7}$ Ferner wird angenommen, daß das Einkommen des ärmsten Fünftels der Bevölkerung vom September 1973 bis September 1980 real um $50 \%$ gesunken ist. ${ }^{8}$ Die Arbeitslosenquote schwankte im Durchschnitt zwischen 13 und $14 \%$. Die vorgenannten Daten muß man im Auge behalten, wenn man zu einer zutreffenden Einschätzung der Reform der Rentenversicherung in Chile kommen will.

\section{Das bisherige Rentenversicherungssystem in Chile}

Die verschiedenen Systeme der sozialen Sicherheit in lateinamerikanischen Ländern weisen fast durchweg ein Merkmal auf, das in einer bedeutenden Studie von Mesa-Lago als "stratification" bezeichnet worden ist. ${ }^{9}$ Mesa-Lagos politisch-soziologische Studie untersucht die Systeme sozialer Sicherheit mehrerer Länder Lateinamerikas unter dem Gesichtspunkt, welche Kräfte die Einrichtung und Entwicklung sowie das Ergebnis der Verteilung von Leistungen sozialer Sicherheit bestimmen. Seine Untersuchung mündet in die These: "A variety of pressure groups with divergent powers constitute the predominant factor in the historical inception of stratified social security system in Latin America and that system generates significant inequalities in the distribution of its services $" .{ }^{10}$ Das heißt, je nach der politisch-sozialen Position einer bestimmten gesellschaftlichen Gruppe variiert der Anteil an und die Versorgung durch Systeme sozialer Sicherheit. In einer Rangfolge hat Mesa-Lago die Militärs, den politisch-administrativen Bereich, den wirtschaftlichen Marktsektor und die Gewerkschaften als einflußreichste gesellschaftliche Kräfte im Bereich der sozialen Sicherheit ausgemacht. Je einflußreicher eine Gruppe ist, desto früher kommt sie in den Genuß sozialer Sicherheit, desto größer

4 Nohlen, D., Chile, in: Nohlen, D./Nuscheler, F. (Hg.), Handbuch der Dritten Welt, Bd. 2, Südamerika: Unterentwicklung und Entwicklung, 2. überarbeitete und ergänzte Ausgabe, Hamburg 1982, S. 214; Rivera, E. (Fn. 2), S. 92.

5 Nohlen, D. (Fn. 4), S. 215.

6 Rivera, E (Fn. 2), S. 83.

7 Rivera, E. (Fn. 2), S. 95.

8 Rivera, E. (Fn. 2), S. 95.

9 Mesa-Lago, C., Social Security in Latin-America, University of Pittsburgh Press 1978.

10 Mesa-Lago, C. (Fn. 9), S. 3. 
ist die Zahl der abgedeckten Risiken, desto großzügiger sind die Leistungen und desto geringer sind die Kosten, die der einzelne für die Finanzierung seiner Leistungen aufzubringen hat.

Chile ist jenes lateinamerikanische Land, das wohl die ausgeprägteste und extremste Form der Schichtung innerhalb des Systems der sozialen Sicherheit aufzuweisen hatte. ${ }^{11}$ So gab es 198018 verschiedene Rentenversicherungsinstitutionen, die jeweils nur für einen bestimmten Versichertenkreis zuständig waren und die sich in Beiträgen und im Leistungsniveau ganz erheblich unterschieden. Zur Illustrierung sollen hier nur die zwei wichtigsten Rentenversicherungsfonds vorgestellt werden. Der Servicio de Seguro Social ist der wichtigste Rentenversicherungsträger für blue-collar-workers. ${ }^{12}$ Die Höhe der Altersrente beträgt in diesem System 50 \% des während der letzten 5 Jahre erzielten Lohnes. Hinzu kommt je $1 \%$ dieses Lohnes für jedes über 10 Beitragsjahre hinaus zurückgelegte weitere Beitragsjahr bis zu maximal 30 Jahren, so daß die Höchstrente $70 \%$ des letzten Durchschnittseinkommens betragen kann. Die Hinterbliebenenrente beträgt $50 \%$ der Rente des Mitgliedes. Bei Invalidenrenten wird zwischen absoluter Invalidität (es können nur noch $30 \%$ der gewöhnlichen Einkünfte erzielt werden) und teilweiser Invalidität (es können mehr als $30 \%$ aber weniger als $60 \%$ der gewöhnlichen Einkünfte erzielt werden) unterschieden.

Bei absoluter Invalidität beträgt die Rente $50 \%$ des Durchschnittseinkommens während der letzten 5 Jahre plus $1 \%$ für jedes über 10 Mitgliedsjahre hinausgehende Beitragsjahr. Die Invalidenrente bei teilweiser Invalidität beträgt die Hälfte der Vollrente.

Die Beiträge werden zu $7 \%$ vom Arbeitnehmer und zu $16 \%$ vom Arbeitgeber, berechnet aus der Lohnsumme, aufgebracht. ${ }^{13}$

Dem gegenüber zeichnet sich EMPART (= Caja de Previsión de Empleados Particolares), der wichtigste Rentenversicherungsträger für white-collar-employees durch ein wesentlich besseres Leistungsniveau aus. ${ }^{14}$ Die Altersrente beträgt 1/35 des während der letzten 5 Jahre erzielten Durchschnittseinkommens für jedes Beitragsjahr, so daß nach 35 Beitragsjahren der Höchstsatz von $100 \%$ erreicht wird.

Ähnlich ist die Invaliditätsrente ausgestaltet. Sie beträgt $70 \%$ des oben bezeichneten Lohnes plus weitere $2 \%$ für jedes das 20 . Beitragsjahr übersteigende Jahr, so daß auch hier nach 35 Jahren $100 \%$ erreicht werden können.

Die Witwenpensionen betragen bei der Alters- und Invaliditätsrente jeweils $50 \%$ der Rente des verstorbenen Versicherten.

Die Ausgestaltung der sozialen Sicherheit in Chile hat zu einem regressiven Einkommensverteilungseffekt zwischen solchen, die nicht versichert sind, und solchen, die versichert sind und innerhalb der letzteren zwischen Gruppen mit niedrigem und hohem Ein-

11 Parrish, Ch. J./Tapía-Videla, J., Welfare Policy and Administration in Chile, in: Journal of Comparative Administration, 1969, S. 455-476.

12 Humeres Magnan, H., Derecho del trabajo y de la seguridad social, 11. Aufl., Santiago 1977, S. 293.

13 Kritzer, B. E., Chile Changes Social Security, in: Social Security Bulletin, Mai 1981, S. 34.

14 Arnold, R., Social Security Reform in Chile, in: Benefits International, 1981, Heft 8, S. 4. 
kommen geführt. Nach einem Bericht der Kommission Prat ${ }^{15}$ haben die Nichtversicherten einen wachsenden Anteil an der Kostenaufbringung für die soziale Sicherheit zu tragen gehabt: $195941 \%, 196544 \%$ und schließlich $197150 \% .{ }^{16}$ Hinzu kam die immer wieder beklagte Ineffektivität der Arbeitsweise der Sozialadministration. So betrugen in Chile die Verwaltungskosten $9 \%$ und waren deshalb 10 mal so hoch wie die Erträge aus den Investitionen. ${ }^{17}$

\section{Die sozialpolitische Konzeption der neoklassischen Wirtschaftstheorie}

Das sozio-ökonomische Modell, mit dessen Hilfe die chilenischen Machthaber eine grundlegende Umgestaltung der chilenischen Wirtschaft angestrebt haben, kann durch die folgenden Elemente charakterisiert werden: Subsidiarität des Staates, Förderung des Privatunternehmers als der tragenden Säule wirtschaftlicher Entwicklung sowie Schaffung eines freien Marktes ohne staatliche Einmischung und Kontrolle. ${ }^{18}$

Demnach ist es nur allzu folgerichtig, daß diese Denkrichtung weitreichende Konsequenzen für die sozialpolitische Intervention des Staates ziehen muß. Wie zu zeigen sein wird, hat das chilenische Arbeits- und Sozialministerium bei der Neugestaltung des Rentenversicherungsrechts expressis verbis auf die Grundannahmen neoklassischer Sozialpolitik zurückgegriffen. Es ist deshalb notwendig, in einem kurzen Uberblick die wesentliche Kritik der neoklassischen Schule an traditioneller Sozialpolitik un die daraus abgeleiteten Reformforderungen darzustellen.

Die herkömmlichen wohlfahrtsstaatlichen Systeme werden als überbeansprucht und überbelastet beschrieben, weil die Regierungen allzu of $t$ dem Einfluß von pressure groups und partikularen Interessen nachgegeben hätten. Als Folge davon seien Initiative, Kreativität und Arbeitsmoral untergraben worden, weil staatliche Leistungssysteme zu viele Ruhekissen geschaffen hätten. ${ }^{19}$

Der zentralste und grundlegendste Einwand der Kritiker des Wohlfahrtsstaates besagt, daß durch diesen die individuelle Freiheit des Bürgers, über sein Glück und Wohlbefinden zu entscheiden, negiert werde. ${ }^{20}$

Die neoklassische Wohlfahrtsökonomie geht davon aus, daß jedes Individuum der beste

15 Dazu Thayer Arteaga, W./Fernandez Florez, E., El nuevo regimen previsional y de cotizaciones, Santiago 1981, S. $35 \mathrm{f}$.

16 Mesa-Lago, C., Seguridad social y probreza, in: CEPAL/PNUD: Se puede superar la pobreza?, Santiago, 1980, S. 174.

17 Mesa-Lago, C. (Fn. 16), S. 179.

$18 \mathrm{Zu}$ einer eingehenden Auseinandersetzung mit neoklassischen Theorien und ihrer Anwendung in Entwicklungsländern vgl. Prebisch, R., The Neoclassical Theories of Economic Liberalism, in: CEPAL-Review, April 1979, S. 167-188.

19 Gilder, G., Wealth and Poverty, New York 1981, S. 112.

20 Hayek, F. A., The Constitution of Liberty, London 1960, S. 261. 
Sachwalter seiner eigenen Wohlfahrt sei, ${ }^{21}$ soziale Wohlfahrt eben gerade von der Wohlfahrt der Individuen abhänge.

Gesamtwirtschaftlich gesehen würden staatliche Systeme sozialer Sicherheit die Sparquote privater Haushalte entscheidend vermindern und damit die Investitionstätigkeit hemmen. Diese ursprünglich ohne Begründung von Friedman ${ }^{22}$ vertretene Auffassung ist in den letzten Jahren vor allem durch Arbeiten von Martin Feldstein auf dem Gebiet staatlicher Altersversorgung eingehend geprüft worden. ${ }^{23}$ Feldstein zufolge führe die Existenz staatlicher Altersversorgung zu einer Halbierung der persönlichen .Sparrate, gleichzeitig werde der verfügbare Kapitalstock reduziert und damit auch das nationale Einkommensniveau entscheidend verschlechtert. ${ }^{24}$ Der Grund sei darin zu sehen, daß Sozialleistungen für alte Menschen nicht aus einem angesparten Kapitalstock erbracht, sondern laufende Leistungen aus laufenden Beiträgen finanziert würden (Umlageverfahren - pay-as-you-go-method).

Des weiteren sei die investitionshemmende Wirkung sozialer Sicherheit der Tatsache geschuldet, daß Beitragsleistungen und Leistungsniveau nicht in einem strengen Konnexverhältnis (Äquivalenzprinzip) stünden. Die Vertreter dieser Auffassung verlangen deshalb die Abschaffung der - wie sie sagen - Sozialversicherungssteuern. ${ }^{25}$

Schließlich wird die Verwaltung des Wohlfahrtsstaates als zu bürokratisch und ineffizient kritisiert. ${ }^{26}$ An die Stelle wohlfahrtsstaatlicher Bürokratie müsse deswegen ein privater Markt von Wohlfahrtsgütern treten, der durch die Konkurrenz verschiedener Anbieter und die individuellen Nutzenentscheidungen der Konsumenten die öffentliche Wohlfahrt bestmöglichst fördere. ${ }^{27}$

\section{Die Position des chilenischen Arbeits- und Sozialministeriums}

In einer Fernsehansprache an das chilenische Volk hat der zuständige Minister für Arbeit und Sozialversicherung die Notwendigkeit der Reform und die Grundlagen des neuen Rechtssystems vorgestellt. ${ }^{28}$ Die Kritik, die der Minister darin an dem alten Rentenversicherungssystem übt, und die Vorzüge, die er in dem neuen System sieht, sind

21 Ausführlich zu diesem Theorem Sugden, R., Hard-luck Stories: Problem of Uninsured in a Laissez-faire Society, in: Journal of Social Policy, 1982, S. 202 ff.

22 Friedman, M., A Theory of the Consumption Function, Princeton University Press, 1957, S. 123.

23 Feldstein, M., Social Security, Induced Retirement, and Aggregate Capital Accumulation, in: Journal of Political Economy, 1974, S. 916; ders., Social Insurance, in: Campbell, C. D. (Hg.), Income Redistribution, Washington D. C., 1977, S. 81-86.

24 Feldstein, M. (Fn. 23), S. 922.

25 Feldstein, M. (Fn. 23), S. 82.

26 Vgl. dazu Bénéton, Ph., Trends in the Social Policy Aims of the United States (1960-1980), in: Labour and Society, 1981, S. $401 \mathrm{ff}$.

27 Zum Thema private Märkte in Welfare Systems s. Higgins, J., Public Welfare: The Road to Freedom?, in: Journal of Social Policy, 1982, S. $186 \mathrm{ff}$.

28 Die Rede ist abgedruckt in: Ministerio del Trabajo y Prevision Social (Hg.), La Reforma Previsional, Santiago 1980, S. 7-22. 
identisch mit der neoklassischen Konzeption sozialer Sicherung, wie sie soeben dargestellt wurde.

Der Minister kritisiert die überkommenen Regelungen, weil sie dem Umlageverfahren (Reparto) unterliegen und den Versicherten eine Sozialversicherungssteuer auferlegen. ${ }^{29}$ Eine strikte Verknüpfung von Beitrag und Leistung sei nicht gegeben.

Uber die Höhe der Sozialversicherungsleistungen entscheide nicht der Beitragszahler, sondern der Gesetzgeber und damit wiederum die jeweils herrschenden politischen Kräfte, da die jeweiligen Regierungen mit ihrem Blick auf die nächsten Wahlen diesen Gruppierungen sich unterwerfen würden. ${ }^{30}$

Das neue Rentensystem folge deshalb dem Kapitalversicherungsverfahren, bei dem die Höhe der Renten von dem durch Beitragszahlungen über die Jahre hinweg angesparten und durch Zinsen vermehrten Kapitalbetrag abhinge. Gefolgt werde einem strikten Äquivalenzprinzip, bei dem die eingezahlten Beiträge genau dem Umfang der Leistungen entsprächen. ${ }^{31}$

Träger der Versicherung sei nicht mehr der Staat, sondern eine Vielzahl privater Verwaltungsgesellschaften (Aktiengesellschaften), die in Konkurrenz zueinander stünden und damit die Effizienz erhöhten. ${ }^{32}$

Der Minister sieht darin eine Garantie zur Wiederherstellung von Effektivität und Stabilität sozialer Sicherung. Die alte staatliche Form der Sozialversicherung wird gleichgesetzt mit Bürokratie und Ineffizienz. ${ }^{33}$

Mit der neuen Regelung werde das bürokratische Dickicht überwunden und die Uberschaubarkeit des Systems hergestellt, weil durch das Äquivalenzprinzip jeder Versicherte wisse, wie hoch aufgrund seiner von ihm erbrachten Beiträge die Leistungen seien. ${ }^{34}$

Besonders deutlich zeigt sich die neoklassische Matrix in der neuen Rentengesetzgebung, wenn der Minister immer wieder darauf hinweist, daß das tragende Leitprinzip des neuen Gesetzes die Verwirklichung des Gedankens der freien Entscheidung des Versicherten sei. ${ }^{35}$ Jeder Versicherte solle entscheiden können, welcher Verwaltungsgesellschaft er angehören wolle. Welche Aktiengesellschaft als effektivste und sicherste anzusehen sei, solle letztlich von der freien Entscheidung und dem Willen der beitragszahlenden Versicherten abhängen.

Fn. 28, S. 7.

30 "Qué gobierno o qué fuerzas politicas con la mirada clavada en futuras elecciones, podían residir las demandas discriminadorias de los grupos con gran poder de presión?", Fn. 28, S. 8.

31 ". . . se logra la indispensable conexión entre esfuerzos y beneficios . ...", Fn. 28, S. 10.

32 Fn. 28, S. $10 \mathrm{f}$.

33 ". . . la historia nos demostró hasta la saciedad que la administración estatal no es garantía ni de seguridad, ni de acciones desinteresadas, como en algún momento se supuso. May por el contrario, en instituciones previsionales dirigidas por quienes no tienen que responder con sus bienes, y más aun en las instituciones con clientes cautivos, casi siempre ha florecido la inefficiencia y la attención inadecuada, con pérdidas para los imponentes y para la sociedad entera", Fn. 28, S. 11.

34 Fn. 28, S. 17.

35 "La reforma previsional recoge en su plenitud uno de los valores que el Gobierno considera crucial en su proyecto de sociedad: la libertad más amplia de elección individual“, Fn. 28, S. 16. 
Mit der Abschaffung der sog. Sozialversicherungssteuer wird - ebenfalls neoklassischem Denken folgend - erwartet, daß die Nachfrage nach Arbeitskräften steigen und damit ein wesentlicher Beitrag zur Investitionstätigkeit und dem Abbau der Arbeitslosigkeit geleistet werde. ${ }^{36}$

\section{Das neue Rentenversicherungssystem}

Die wesentliche Rechtsgrundlage, mit der das neue Rentensystem eingeführt wurde, ist das Decreto Ley Nr. 3500 (im folgenden = D. L. 3500) vom 13. 11. 1980.37

\section{Kreis der Versicherten}

Gemäß Art. 2 D. L. 3500 führt die Begründung eines Arbeitsverhältnisses zur automatischen Mitgliedschaft in der neuen Rentenversicherung und zur Verpflichtung, Beiträge an eine Verwaltungsgesellschaft (Administradora) i. S. des Gesetzes zu zahlen. Der Arbeitnehmer ist deshalb verpflichtet, seinem Arbeitgeber Mitteilung darüber zu machen, in welchem Versicherungsunternehmen er versichert sein will und der Arbeitgeber hat den Beginn (ebenso wie das Ende) des Arbeitsverhältnisses der jeweiligen Verwaltungsgesellschaft anzuzeigen (Art. 2 Abs. 5 u. 6 D. L. 3500).

Neben den abhängig Beschäftigten haben gemäß Art. 89 D. L. 3500 Selbständige die Berechtigung, freiwillig der neuen Rentenversicherung beizutreten. Die Mitgliedschaft entsteht mit der ersten Beitragszahlung (Art. 89 Abs. 2 D. L. 3500).

Diejenigen Arbeitnehmer, welche nach bisherigem Recht Beiträge zu einer Sozialversicherungsinstitution leisten, (oder geleistet haben), haben ein Wahlrecht, ob sie in ihrer bisherigen Versicherung verbleiben oder sich dem neuen System anschließen wollen (Titel XI, Art. 1 Abs. 1 D. L. 3500). Dieses Wahlrecht besteht bis einschließlich 31. 12. 1982 für solche Personen, die erstmals ein Arbeitsverhältnis eingehen (Titel XI, Art. 1 Abs. 2 D. L. 3500). Die Begründung einer Rentenversicherung nach neuem Recht kann frühestens mit Wirkung vom 1.5.1989 an und spätestens mit Wirkung vom 1. 5.1986 an erfolgen (Titel XI, Art. 1 Abs. 3 D. L. 3500).

Um die Entscheidung für den Ưbergang zur neuen Regelung zu erleichtern, räumt das Gesetz dem Arbeitnehmer ein Guthaben aus der alten Rentenversicherung (Bono de Reconocimiento) ein, das den bisherigen Beitragszeiten entspricht (Titel XI, Art. 3 D. L. 3500). Voraussetzung für diesen Bonus ist allerdings, daß in den letzten 5 Jahren, die der Veröffentlichung des D. L. 3500 vorausgehen, mindestens 12 Beiträge zur Rentenversicherung geleistet worden sind (Titel XI, Art. 4 Abs. 1 D. L. 3500). Die Höhe des Bono de Reconociemento errechnet sich wie folgt (Titel XI, Art. 4 D. L. 3500): zunächst ist ein Betrag in Höhe von 80 \% der Summe der Einkünfte festzustellen, die als Grundla-

36 Fn. 28 , S. $20 \mathrm{f}$.

37 Der Gesetzestext ist abgedruckt in: Ministerio del Trabajo y Prevision Social (Fn. 28), S. 89-133. 
ge für die letzten 12 Monatsbeiträge, die vor dem 30. 6. 1979 geleistet wurden, gedient haben. Der so ermittelte Betrag ist mit einem Quotienten zu multiplizieren, der sich aus der Division der Zahl der Beitragsjahre durch die Zahl 35 ergibt. Ist dieser Quotient höher als 1, so darf die Multiplikation dennoch nur mit der Zahl 1 erfolgen. Das Produkt ist mit 10,35 zu multiplizieren, wenn es sich um ein männliches Mitglied handelt, und mit 11,36 bei Frauen. ${ }^{38}$

\section{Finanzierung}

Das neue chilenische Rentenversicherungsrecht folgt dem Kapitalisierungsprinzip. Grundlage für die Finanzierung der Alters-, Invaliditäts- und Hinterbliebenenrenten ist das von den Versicherten angesparte Kapital. Die versicherten Arbeitnehmer sind zu diesem Zwecke verpflichtet, $10 \%$ ihrer Einkünfte auf ein Kapitalkonto einzuzahlen (Art. 17 D. L. 3500). Was unter Einkünften zu verstehen ist, wird aufgrund des Verweises in Art. 14 D. L. 3500 durch Art. 50 des D. L. 2200 vom 15. 6.1978 bestimmt.

Nach der darin enthaltenen Definition handelt es sich im wesentlichen um die vom Arbeitgeber erbrachten Geldleistungen sowie Naturalleistungen, die in Geld bewertbar sind. Selbständige haben ebenfalls $10 \%$ ihres beitragspflichtigen Einkommens zu zahlen (Art. 15, 17 D. L. 3500). Gemäß Art. 90 D. L. 3500 hat jeder versicherte Selbständige jeden Monat sein monatliches beitragspflichtiges Einkommen dem Versicherungsunternehmen mitzuteilen, wobei ein gewisser Mindestbetrag nicht unterschritten und ein Höchstbetrag von 120 Unidades de Fomento ${ }^{40}$ nicht unterschritten werden darf.

Jeder Versicherte muß ferner einen bestimmten Prozentsatz seiner Einkünfte als Beitrag zur Finanzierung der Invaliditäts- und Hinterbliebenenrenten, die während seines aktiven Versicherungslebens entstehen können, zahlen (Art. 18 Abs. 1 D. L. 3500). Die Beitragshöhe wird von jeder Verwaltungsgesellschaft eigenständig festgesetzt und muß öffentlich bekannt gegeben werden (Art. 18 Abs. 2 D. L. 3500). Bei der Festlegung der Beitragshöhe dürfen nur folgende Faktoren berücksichtigt werden (Art. 18 Abs. 2 und D. L. 3500): Saldo des individuellen Rentenkontos dividiert durch die in Art. 52 D. L. 3500 bestimmte Versicherungssumme, Alter der potentiellen Anspruchinhaber der Hinterbliebenenrente, Alter des Versicherten und der in Art. 52 D. L. 3500 genannte Prozentsatz zur Bestimmung der Versicherungssumme. ${ }^{41}$

Gemäß Art. 19 D. L. 3500 ist jeder Arbeitgeber verpflichtet, die nach diesem Gesetz bestehenden Versicherungsbeiträge vom Lohn einzubehalten und an die Verwaltungsge-

38 Zu weiteren Einzelf ragen Thayer Arteaga, W./Fernandez Florez, E. (Fn. 15), S. 187-198.

39 Erhält ein Versicherter Krankengeld (subsidio de incapacidad laboral) gem. D.F.L. no. 44 aus dem Jahre 1978, so hat während des Bezuges des Krankengeldes die zahlende Behörde die Beiträge an die Verwaltungsgesellschaft abzuführen.

40 Chile hat eine sog. "indexed economy", d. h. alle finanziellen Transaktionen werden in Einheiten statt in Geldwerten ausgedrückt.

41 Für eine Úbergangszeit von zwei Jahren können die Verwaltungsgesellschaften gem. Art. 16 der Ubergangsvorschrift des D.L. 3500 Beiträge in Höhe von 3 \% des Lohnes erheben und diese an die Versicherungsgesellschaft abführen. Danach müssen feste Tarife bestimmt werden. 
sellschaft innerhalb von 10 Tagen des Monats, der auf den folgt, in dem die beitragspflichtigen Einkünfte fällig werden, abzuführen.

Wird das Arbeitsverhältnis beendet oder suspendiert, so können versicherte Arbeitnehmer freiwillig bis zu einem Jahr Beiträge leisten, um Anrechte auf Invaliditäts- und Hinterbliebenenleistungen zu erhalten (Art. 20 Abs. 1 D. L. 3500), vorausgesetzt er tut seine Entscheidungen in dieser Richtung durch Zahlung eines freiwilligen Beitrages innerhalb von 30 Tagen nach Zahlung eines obligatorischen Beitrages kund (Art. 20 Abs. 2 D. L. 3500). Aus der Formulierung des Art. 20 D. L. 3500 ist zu schließen, daß ganz offensichtlich nach Beendigung eines Arbeitsverhältnisses keine Möglichkeit der Aufrechterhaltung des Versicherungsschutzes für den Versicherungsfall Alter besteht.

Eine Höherversicherung sieht Art. 21 D. L. 3500 vor. Uber die Pflichtbeiträge hinaus können weitere $10 \%$ des beitragspflichtigen Einkommens geleistet werden und weitere $20 \%$ des Betrages, der das beitragspflichtige Einkommen übersteigt, allerdings nach oben begrenzt durch 120 Unidades de Fomento.

\section{Das Rentenleistungssystem}

\subsection{Altersrente}

Anspruch auf Altersrente hat der männliche Versicherte, der das 65. Lebensjahr vollendet hat, und der weibliche Versicherte, der das 60. Lebensjahr vollendet hat (Art. 3 D. L. 3500).

Sind diese Voraussetzungen erfüllt, so hat der Versicherte ein Wahlrecht, sein Versicherungskonto zur Begründung einer Altersrente einzusetzen (Art. 62 D. L. 3500):

a) er kann mit seinem Guthaben bei einer privaten Versicherungsgesellschaft eine Leibrente (renta vitalicia) ${ }^{42}$ erwerben, aus der ihm monatliche Zahlungen bis zu seinem Tode erwachsen. In diesem Falle transferiert die Verwaltungsgesellschaft die nötige Geldmenge unmittelbar an die Versicherungsgesellschaft.

b) Der Versicherte kann sein Rentenkonto beider Verwaltungsgesellschaft belassen und dafür ein monatliches Ruhegeld beziehen.

\section{zu a)}

Die Modalitäten dieser Versicherungsart werden im wesentlichen von der Aufsichtsbehörde (dazu unten) festgelegt (Art. 63 D. L. 3500). In jedem Fall muß der Versicherte von seinem angesparten Kapital soviel für die Leibrente einsetzen, daß sie nach Möglichkeit $70 \%$ der in den letzten 10 Jahren erzielten beitragspflichtigen Durchschnittseinkünfte erreicht und eventuelle Hinterbliebenenrenten gewährleistet (Art. 64 Abs. 1 D. L. 3500). Sind diese Voraussetzungen erfüllt, so kann der Versicherte über den Restbetrag frei verfügen.

42 Der Leibrentenvertrag ist in Art. 2264 Código Civil geregelt. 


\section{zu b)}

Wählt der Versicherte diese Alternative, so erhält er eine Jahresrente, deren Betrag sich ergibt, wenn man den effektiven Saldo des Rentenkontos durch die Zahl dividiert, die sich aufgrund der Lebenserwartung der familiären Gruppe (grupo familiar) ergibt (Art. 66 Abs. 1 D. L. 3500). ${ }^{43}$

Die Möglichkeit einer vorgezogenen Altersrente, d. h. vor Erreichen der in Art. 3 D. L. 3500 genannten Altersgrenze, sieht Art. 71 D. L. 3500 unter bestimmten Voraussetzungen vor.

\subsection{Invaliditätsrente}

Der Anspruch auf Invalidenrente setzt gemäß Art. 4 D. L. 3500 voraus, daß ein Anspruch auf Altersrente nicht besteht und infolge von Krankheit oder Schwächung der körperlichen oder geistigen Kräfte zwei Drittel der Arbeitsfähigkeit verloren gegangen sind.

Das letztgenannte Tatbestandsmerkmal muß von einer Kommission bestätigt werden, die aus drei Chirurgen besteht und für jede Region von der Aufsichtsbehörde ernannt wird (Art. 11 Abs. 1 D. L. 3500). Die Entscheidungen dieser Kommission können bei dem für das Departement örtlich zuständigen Arbeitsgericht oder soweit ein Departement nicht vorhanden ist, bei dem sog. Juez de Letras angegriffen werden.

Die Höhe der Invaliditätsrente ergibt sich aus den Art. 51, 52 D. L. 3500. Sie entspricht einer bestimmten Versicherungssumme (ingreso cubierto por el seguro), welche jede Verwaltungsgesellschaft bei einer Privatversicherung gemäß Art. 58 D. L. 3500 zu versichern hat und wozu sie die über Art. 18 D. L. 3500 zu erhebenden Beiträge einzusetzen hat. Diese Versicherungssumme ist der prozentuale Anteil an einem sog. Grundbetrag (ingreso base), dessen Höhe bestimmt ist durch die durchschnittlichen beitragspflichtigen Einkünfte während der letzten 12 Monate (Art. 52 Abs. 1, Abs. 2 D. L. 3500). Der vorgenannte Prozentsatz hängt davon ab, wie lange jemand Beiträge zu einer Sozialversicherungsinstitution geleistet hat. Bei 5 Jahren Beitragszeit oder darunter beträgt die Invaliditätsversicherungssumme $50 \%$ des Grundbetrages, für jeden weiteren Fünfjahreszeitraum erhöht sich der Prozentsatz um weitere $10 \%$ bis zu maximal $70 \%$ des Grundbetrages (Art. 52 Abs. 3, Abs. 4 D. L. 3500). Nach Art. 52 Abs. 5 D. L. 3500 können Versicherte eine höhere Versicherungssumme bestimmen (müssen dafür aber natürlich höhere Versicherungsbeiträge in Kauf nehmen).

\section{3. Hinterbliebenenrente}

Der Kreis der Anspruchsberechtigten ergibt sich aus Art. 5 ff. D. L. 3500. Es sind dies alle Mitglieder der Familiengruppe des Verstorbenen (componentes del grupo familiar),

Die Legaldefinition des Begriffs der familiären Gruppe ist in Art. 68 D.L. 3500 enthalten. 
worunter man den überlebenden Ehegatten, die ehelichen, nichtehelichen und Adoptivkinder, die Eltern sowie die Mutter der nichtehelichen Kinder des Verstorbenen versteht, immer vorausgesetzt, daß kein Anspruch auf eine andere Sozialversicherungsrente besteht.

Die Witwe muß, um einen Anspruch zu haben, ihre Ehe mit dem Verstorbenen mindestens 6 Monate vor dessen Tod abgeschlossen haben bzw. 3 Jahre vor dem Tod, wenn der Verstorbene im Zeitpunkt der Ehe eine Alters- oder Invaliditätsrente erhielt (Art. 6 Abs. 1 D. L. 3500). Diese Einschränkungen gelten aber nicht, wenn im Zeitpunkt des Todes die Witwe schwanger ist oder gemeinsame minderjährige Kinder vorhanden sind. Ein Witwer erhält nur dann Hinterbliebenenrente, wenn er im Zeitpunkt des Todes seiner Ehefrau Invalide im Sinne des Art. 4 D. L. 3500 ist (Art. 7 D. L. 3500).

Die Höhe der Hinterbliebenenrente hängt davon ab, ob ihre Entstehung in die Zeit der aktiven Mitgliedschaft des versicherten Verstorbenen fällt (1) oder in die Zeit, in der der Versicherte bereits Altersrente erhielt (2).

\section{zu (1)}

Die Höhe der Hinterbliebenenrenten berechnet sich ebenfalls nach den Art. 51, 52 D. L. 3500, und es gilt für den Berechnungsmodus analog das, was oben zur Invaliditätsrente ausgeführt wurde. Auch hier begegnet man also wieder dem grundlegenden Finanzierungsmechanismus der Privatversicherung, wie er in dem D. L. 3500 angelegt ist. Die Versicherten müssen Beiträge zur Finanzierung von Hinterbliebenenrenten leisten, wobei ebenso wie bei der Invaliditätsrente eine bestimmte Versicherungssumme abzudekken ist. Die Hinterbliebenenrente ist ein prozentualer Anteil an dieser Versicherungssumme und ist für die verschiedenen anspruchsberechtigten Personen unterschiedlich gestaltet (vgl. Art. 54 D. L. 3500).

zu (2)

Stirbt der Bezieher einer Altersrente, so hängen Art und Umfang der Hinterbliebenenrente davon ab, welche Form der Altersrente der Verstorbene gewählt hatte.

Hatte er sich für eine Leibrentenversicherung gemäß Art. 62 Nr. 1 D. L. 3500 entschieden, so ist gleichzeitig damit für die Hinterbliebenen Sorge getragen. Denn es ist vorgeschrieben, daß beim Abschluß eines Leibrentenvertrages die Hinterbliebenen zu berücksichtigen sind.

War dagegen eine Altersrente nach Art. 62 Nr. 2 D. L. 3500 gewählt worden, so bestimmt sich die Hinterbliebenenrente nach Art. 72 D. L. 3500. Die Hinterbliebenenrente unterliegt in diesem Falle dem gleichen Berechnungsschema, das für die Altersrente gemäß Art. 66, 68 D. L. 3500 anzuwenden war (s. o.).

Sind keine anspruchsberechtigten Hinterbliebenen vorhanden, so fällt der Saldo des Versicherungskontos in den Nachlaß (Art. 72 Abs. 3 D. L. 3500). 


\subsection{Gesetzliche Mindestrenten}

In Abweichung von dem im übrigen strikt eingehaltenen Grundsatz der Äquivalenz von Beitrag und Leistung gewährt der Staat unter bestimmten Voraussetzungen Mindestrenten.

Bezüglich der Mindestaltersrente verweist Art. 73 Abs. 2 D. L. 3500 auf Art. 26 des Gesetzes Nr. 15386 vom 11. 12. 1963, wo bestimmt ist, daß die Mindestalters- und -invaliditätsrente $85 \%$ des industriellen Mindestlohns beträgt.

Reicht das durch eigene Beiträge angesparte Kapital zur Begründung einer Rente in Höhe der soeben bezeichneten Mindestrente nicht aus, so wird der Differenzbetrag durch einen staatlichen Zuschuß gedeckt.

Anspruch auf die Mindestaltersrente haben alle Versicherten, welche die in Art. 3 D. L. 3500 vorgeschriebene gesetzliche Altersgrenze erreicht haben und außerdem mindestens 20 Jahre Beiträge nach den für ihre Rentenversicherung einschlägigen Vorschriften geleistet haben (Art. 75 D. L. 3500).

Die Voraussetzungen für die Mindestrente bei Invalidität sind im einzelnen in Art. 77 D. L. 3500 genannt.

Die Mindestrente für Hinterbliebene sind bestimmte Prozentbeträge der Mindestaltersrente, die je nach der Person des Hinterbliebenen variieren (vgl. Art. 78 D. L. 3500).

\section{Träger der Versicherung}

Träger des neuen Rentenversicherungssystems sind Verwaltungsgesellschaften (Administradoras) in der Rechtsform von Aktiengesellschaften, deren einzige Aufgabe die Verwaltung eines Rentenfonds sowie die Gewährung von Leistungen nach diesem Gesetz sind (Art. 23 D. L. 3500).

Das gesetzlich festgelegte Grundkapital dieser Aktiengesellschaften beträgt 20000 Unidades de Fomento (Art. 24 D. L. 3500). Art. 26 D. L. 3500 statuiert die Informationspflichten der Verwaltungsgesellschaft für die Offentlichkeit, welche das Kapital, die Investitionen, die Rentabilität etc. betreffen.

Ein wesentliches Anliegen des Gesetzgebers war es, die Rentenversicherung durch eine Vielzahl von Trägern durchzuführen, um einen intensiven Wettbewerb zu gewährleisten. Dazu war es nötig, den Versicherten die freie Entscheidung über die Wahl einer bestimmten Verwaltungsgesellschaft zu überlassen und einen jederzeitigen Wechsel zwischen den verschiedenen Gesellschaften zuzulassen. Diese Möglichkeit wurde durch Art. 32 D. L. 3500 eingeräumt, wonach der Versicherte seinen Anteil auf eine andere Verwaltungsgesellschaft übertragen kann, wenn er 30 Tage zuvor seiner bisherigen Verwaltungsgesellschaft und dem Arbeitgeber Mitteilung davon gemacht hat.

Die von den Verwaltungsgesellschaften verwalteten Fonds stellen ein unabhängiges und von dem Vermögen der Verwaltungsgesellschaft getrenntes Sondervermögen dar (Art. 33 Abs. 1 D. L. 3500), das sich aus den Beiträgen, Säumniszuschlägen etc., den Er- 
trägen aus den Investitionen abzüglich der Bearbeitungsgebühren der Verwaltungsgesellschaft, zusammensetzt (Art. 33 Abs. 2 D. L. 3500).

Der Wert eines Rentenfonds wird ausgedrückt in gleichen Werteinheiten, wobei eine Werteinheit täglich bestimmt wird auf der Basis des Wertes der getätigten Investitionen (Art. 35 D. L. 3500). Die Art der Investitionen wird durch Art. 44, 45 D. L. 3500 festgelegt. Etwas vereinfacht ausgesprochen werden Investitionen in "low-risk"-Anlagebereichen verlangt.

Das Gesetz legt den Versicherungsunternehmen die Verpflichtung auf, dafür zu sorgen, daß der Rentenfonds einen monatlichen Mindestprofit (rentabilidad promedio minima) erzielt (Art. 37 D. L. 3500). ${ }^{44}$ Zur Sicherung des Mindestprofits müßten die Verwaltungsgesellschaften eine Fluktuationsreserve (Art. 38, 39 D. L. 3500) und eine Kassenreserve bilden (Art. 40 D. L. 3500).

Dem Wettbewerbsgedanken entsprechend muß eine Verwaltungsgesellschaft aufgelöst werden, wenn es ihr nicht gelingt, eine monatliche Mindestdurchschnittsrendite zu erzielen. Im einzelnen sieht das Auflösungsverfahren wie folgt aus (Art. 42 D. L. 3500):

Bleibt der Ertrag des Rentenfonds einen Monat lang unterhalb der Mindestgrenze und kann der Differenzbetrag nicht mit den Mitteln der Fluktuationsreserve ausgeglichen werden, so hat die Verwaltungsgesellschaft innerhalb von 5 Tagen eine Deckung des Betrags mit Mitteln der Kassenreserve vorzunehmen, die aber innerhalb von 2 Wochen zurückgeführt werden müssen.

Gelingt es nicht, so zahlt der Staat den Differenzbetrag. In diesem Falle leitet der Arbeits- und Sozialminister die Liquidation der AG ein, wobei dem Staat im Hinblick auf seine Ausgleichszahlungen ein Vorzugsrecht eingeräumt wird. Die Versicherten müssen sich innerhalb von 90 Tagen nach der Liquidation bei einer anderen Verwaltungsgesellschaft einschreiben. Beim Abschluß der Liquidation, die von der Aufsichtsbehörde durchgeführt wird, werden die Anteile der Versicherten auf die jeweiligen Konten der Verwaltungsgesellschaft übertragen, bei denen sich die Versicherten neu eingeschrieben haben (Art. 43 D. L. 3500).

\section{Versicherungsauf sicht}

Die Versicherungsaufsicht obliegt einer Behörde (vom Gesetz als Superintendencia de Administradoras de Fondos de Pensiones bezeichnet), einer selbständigen juristischen Person mit eigenem Vermögen (Art. 93 D. L. 3500). Ihre wesentlichen Aufgaben sind (Art. 94 D. L. 3500): Genehmigung von Verwaltungsgesellschaften, Entscheidung über Streitigkeiten betreffend die Auslegung des Gesetzes, Uberwachung der Tätigkeit der Verwaltungsgesellschaften, insbesondere ihres Finanzgebarens, Aufstellung von Versicherungsbedingungen, Abschluß von nach diesem Gesetz vorgesehenen Versicherungsverträgen, Auferlegen von Geldbußen, sowie die Liquidation von Versicherungsunternehmen.

Weitere Einzelheiten über die Einrichtung, Ausstattung und Aufgaben der superintendencia sind in dem Decreto con Fuerza de Ley No. 101 vom 29. November 1980 enthalten, das aufgrund der Ermächtigung in Art. 93 D. L. 3500 ergangen ist. 


\section{Kritik der Rentenversicherungsref orm}

Die Kritik an der chilenischen Rentenversicherungsreform soll nicht auf einer abstrakten Ebene erfolgen, d. h. in einer von den nationalen Besonderheiten absehenden Diskussion über das Für und Wider von öffentlichen Sozialversicherungssystemen einerseits und privaten Sparmodellen andererseits. ${ }^{45}$ Vielmehr geht es im folgenden darum, die chilenische Rentenreform in ihrem konkreten Bezug zur gegenwärtigen gesellschaftlichen Situation des Landes zu bewerten.

Dabei empfiehlt es sich, vorweg als Prüfungsmaßstab die eigenen Prämissen und Ziele zu wählen, die der chilenische Gesetzgeber an die Reform geknüpft hat. Hauptidee der Reform war, die Entscheidungsfreiheit des chilenischen Bürgers zu sichern, "freedom to choose" war die oberste Losung. Bei genauerem Hinsehen zeigt sich jedoch, daß sich hinter dieser Formel wenig Substrat verbirgt. Zum einen ist die Mitgliedschaft in der Rentenversicherung obligatorisch. Eine Wahlfreiheit für ein anderes, privates Rentenmodell besteht nicht. Die Wahlfreiheit der Versicherten beschränkt sich vielmehr auf die Bestimmungen der Verwaltungsgesellschaft, wobei ausschlaggebend für die Entscheidung die Effektivität der Gesellschaft, d. h. die erreichte Verzinsung des angelegten Kapitals, sein soll. Dies würde aber voraussetzen, daß die Versicherten auch wirklich in der Lage sind, eine solche Entscheidung zu treffen. Verlangt würde demnach eine Anlegermentalität, die, ausgestattet mit den nötigen Informationen über die Arbeitsweise und die Erfolge der einzelnen Verwaltungsgesellschaften, ein höchstmögliches Anlageoptimum erzielt. Daß derartige Voraussetzungen für die Mehrheit der Versicherten in Chile bestehen, dürfte wohl von kaum jemandem behauptet werden.

Eine weitere denkbare Möglichkeit individueller Entscheidung der Versicherten eröffnet der Anspruch auf vorzeitiges Altersruhegeld. Die Voraussetzungen dazu (vgl. Art. 71 D. L. 3500) sind allerdings nicht leicht zu erfüllen, da eine bestimmte Rentenhöhe vorausgesetzt wird, die für den Durchschnittsverdiener kaum zu erreichen sein dürfte. Die vorgezogene Altersrente wird deshalb für die Mehrheit der Versicherten nur auf dem Papier bestehen, in ihren Genuß dürften wohl nur Höherverdiener kommen.

Schließlich bleibt als Wahlmöglichkeit die Entscheidung darüber, ob als Altersruhegeld eine Leibrente gem. Art. 62 Ziff. 1 D. L. 3500 oder periodische Rentenzahlungen aus dem eigenen Rentenkonto nach Art. 62 Ziff. 2 D. L. 3500 erfolgen soll. Auch hierbei stellt sich erneut die Frage, ob die Mehrheit der Versicherten in der Lage sein wird, die Vorzüge der verschiedenen Modelle zu durchschauen und eine optimale Entscheidung zu treffen. Die Kriterien für die Beurteilung der einzelnen Modelle sind nämlich nicht generell zu bestimmen, sondern hängen von den individuellen Lebensumständen der Versicherten $a b .{ }^{46}$ Eine wirkliche Wahl werden nur diejenigen treffen können, die mit dem Sy-

45 S. dazu die Beiträge in: Von Furstenberg, G. M. (Hg.), Social Security Versus Private Saving, Cambridge (Mass.), 1979.

46 Thayer Arteaga, W./Fernandez Florez, E. (Fn. 15), S. 142 f. 
stem gut vertraut sind und die dahinter steckenden Versicherungsmechanismen erfassen können.

So muß im Hinblick auf das Prinzip der Wahl- und Entscheidungsfreiheit das Urteil über die Reform negativ ausfallen. Die Problematik wird im übrigen auch von solchen Autoren gesehen, die der Reform positiv gegenüberstehen. So schreibt Arnold:47 "The issues involved are quite profound and the average Chilean may not be fully aware of the elements involved in making a reasoned choice". Arnold will diesen Schwierigkeiten dadurch begegnen, daß er an die Arbeitgeber appelliert, die nötige Aufklärungsarbeit zu leisten. ${ }^{48}$ Damit würde aber indirekt anerkannt, daß offensichtlich die zentrale Figur für die Reform, nämlich der homo oeconomicus, in der Realität weitgehend fehlt. Die Verantwortung für die Realisierung der Reform aber einem Dritten, hier dem Arbeitgeber, aufzuerlegen, widerspricht den grundsätzlichen Annahmen der neoklassischen Wohlfahrtstheorie, nämlich der Annahme, daß jeder einzelne für die Optimierung seiner Wohlfahrt verantwortlich ist (best judge principle).

Ein weiteres Ziel der chilenischen Rentenreform war es, die Beitrags- und Leistungsgerechtigkeit zu vermehren und das Leistungsniveau zu verbessern. Demnach wäre es an sich darum gegangen, die bestehenden Privilegien auf der Beitrags- und Leistungsseite abzubauen. Dem widerspricht jedoch, daß das neue System wichtige Gruppen wie Militär und Polizei nicht einbezogen hat, die bislang eine optimale soziale Sicherung genossen haben. Sie gehören weiterhin der Caja de Previsión de la Defensa Nacional und der Dirección de Previsión de Carabinieros de Chile an. Verteidigt wurde die Beibehaltung dieser Systeme damit, daß die Besonderheit der beruflichen Tätigkeit ihrer Mitglieder noch intensive Studien erforderten, um entscheiden zu können, ob auch sie in das allgemeine System einbezogen werden könnten. ${ }^{49}$

Auf der anderen Seite hat das Gesetz die alten Privilegien dadurch aufrechterhalten, daß es eine Option für den Verbleib in den alten Rentenfonds gewährt hat, ohne diese Systeme selbst zu verändern. Demnach werden wohl alle diejenigen in den alten Systemen verbleiben, denen nach der bisherigen Gesetzgebung Privilegien eingeräumt worden sind.

Mit der neuen Regelung wurde die strikte Anwendung des Äquivalenzprinzips eingeführt, d. h. Beiträge und Leistungen entsprechen einander. Damit wurde ganz ohne Zweifel ein Beitrag im Sinne einer iustitia commutativa geleistet. Was dies allerdings auf dem Hintergrund der gegenwärtigen Wirtschafts- und Sozialstruktur Chiles bedeutet, muß man sich anhand folgender Uberlegungen und Daten vor Augen führen. Wie schon oben ausgeführt, hat die jüngste chilenische Entwicklung zu einer weiteren Vertiefung des schon früher bestehenden hohen Einkommensgefälles geführt. ${ }^{50}$ Das heißt von der

47 Arnold, R. (Fn. 14), S. 6.

48 Arnold, R. (Fn. 14), S. 6: "Perhaps the onus should be on employers to make presentations outlining the salient points".

49 Ministerio del Trabajo y Prevision Social (Fn. 28), S. 53.

50 S. dazu Tabelle bei Rivera, E. (Fn. 2), S. 95. 
Sozialversicherungsreform profitieren die Höherverdienenden, eine Umverteilung zugunsten Minderbemittelter findet nicht statt.

$17 \%$ der chilenischen Bevölkerung leben unter der Armutsgrenze (poverty line). ${ }^{51}$ Soweit diese Gruppen überhaupt in einem Arbeitsverhältnis stehen und damit die Versicherteneigenschaft begründen können, ist klar, daß ihre Beiträge keine nennenswerten Rentenleistungen hervorbringen können. In diesem Zusammenhang muß auch die Einbeziehung der Selbständigen in das neue Rentensystem relativiert werden, die von dem chilenischen Gesetzgeber immer wieder als besondere Errungenschaft hervorgehoben worden ist. In der Tat ist die soziale Sicherheit Selbständiger in Entwicklungsländern ein gravierendes Problem, um dessen Lösung sich vor allem internationale Organisationen immer wieder in Zusammenarbeit mit den Länderregierungen bemüht haben..$^{52} \mathrm{Im}$ Kontext von Entwicklungsländern darf man bei der Kategorie der Selbständigen nicht vornehmlich an die freien Berufe, sondern an das unzählige Heer von Kleinhändlern, Handwerkern und own-account-workers denken, deren finanzielle und soziale Lage vielfach sehr bedrückend ist. Ihre Lage wird man aber sicherlich nicht dadurch verbessern können, daß man ihnen die Möglichkeit zum Beitritt in ein streng nach dem Äquivalenzprinzip funktionierendes Versicherungssystem gestattet. Ihr Einkommen wird es häufig nicht zulassen, Versicherungsbeiträge zu erbringen bzw. solche Beiträge, die einen Anspruch auf bedarfsdeckende Leistungen geben.

Ein weiteres gewichtiges Moment, dem die neue Reform der Rentenversicherung in Chile überhaupt nicht Rechnung trägt, ist die hohe Arbeitslosenquote. Im Jahre 1981 betrug die Arbeitslosigkeit $14 \% .{ }^{53}$ Im Juni 1982 ist die Zahl der Arbeitslosen auf $18 \%$ angestiegen. ${ }^{54}$ Soweit es sich dabei um Jugendliche oder andere Arbeitslose handelt, die noch nie einen Arbeitsplatz gehabt haben, kommen sie ohnehin nicht in den Anwendungsbereich des D. L. 3500. Aber auch für arbeitslos gewordene ehemalige Versicherte bietet die Reform keine ernsthafte Lösung an. Zwar haben diese über Art. 20 D. L. 3500 die Möglichkeit, freiwillig Beiträge zu leisten. Allerdings besteht diese Möglichkeit nur bis längstens zu einem Jahr und nur um Anrechte auf Invaliditäts- und Hinterbliebenenrenten zu zielen. Es gibt zwar eine Arbeitslosenunterstützung (subsidio de cesantia), die jedoch nur $75 \%$ des letzten Lohnes beträgt und nur für 6 Monate gewährt wird (vgl. Art. 8 D. L. 603 vom 10. 8. 1974). Von der Ermessensvorschrift, eine Zahlung bis zu einem Jahr fortzusetzen, macht die Praxis nicht Gebrauch. Es dürfte deshalb dem einzelnen arbeitslos gewordenen Arbeitnehmer nur sehr schwer fallen, seine Standardbeiträge in der Rentenversicherung aufzubringen.

Unberücksichtigt ist weiter das Problem der Diskontinuität der Arbeitsverhältnisse ge-

51 Nachweise bei Altimir, O., Poverty, Growth and Basic Needs in Different Value Systems, in: CEPAL Review, April 1981, S. 74.

52 Zu den Problemen bei der Einbeziehung Selbständiger vgl. Bureau International du Travail, Rapport concernant la planification des régimes de sécurité sociale (invalidité, vieillesse, survivants) pour les travailleurs indépendants en Tunisie, Genf 1980.

53 Arnold, R. (Fn. 14), S. 2.

54 Süddeutsche Zeitung vom 21. 6. 1982, S. 7. 
blieben. Gelegenheitsarbeit ist in Chile weit verbreitet und die damit verbundenen Schwierigkeiten verwaltungsgemäßer Art (Anmeldung, ordentliche Abführung von Beiträgen) sowie das geringe Beitragsniveau (angesichts geringer Entlohnung) sind eine schwere Hypothek, für die ein privates Versicherungsmodell mit Äquivalenzprinzip keine Lösungen bereithält.

Ebenfalls außer Acht gelassen ist die Situation von mitarbeitenden Familienangehörigen, die häufig nicht entlohnt werden und deshalb natürlich für irgendwelche Beiträge nicht aufkommen können.

An der Grundeigenschaft des überkommenen Versicherungssystems in Chile, nämlich seiner pyramidenförmigen Ausgestaltung, wodurch im System nur die allgemeinen Einkommensunterschiede widergespiegelt werden, hat sich durch die Reform nichts geändert. Mit ihr wurde lediglich ein Zwangssparsystem normiert, von dem erwartungsgemäß nur Gehalts- und Lohnempfänger mit regelmäßigen Einkommen profitieren können, dessen Höhe das Ansparen ausreichenden Kapitals zuläßt. Da jeder Umverteilungsmechanismus ausgeschlossen ist, insbesondere das wesentliche Element der Risikoverteilung (risk-pooling) fehlt, ist für das neue chilenische System der Terminus soziale Sicherheit nicht angebracht..$^{55}$

Der Vorwurf der Nichtberücksichtigung der wirtschaftlichen und sozialen Verhältnisse in Chile durch die neue Rentenversicherungsreform gilt vor allem im Verhältnis zur Landbevölkerung. Die Vernachlässigung der Landbevölkerung, vor allem der in der Landwirtschaft Tätigen, durch Systeme sozialer Sicherheit ist in den vergangenen Jahren zu einem, vielleicht zu dem zentralen Thema in den Bemühungen internationaler Organisationen, vor allem des Internationalen Arbeitsamtes und der Internationalen Vereinigung für soziale Sicherheit in Genf geworden. ${ }^{56}$ Man hat erkannt, daß einerseits die Bedürftigkeit der Landbevölkerung besonders groß ist, andererseits die herkömmlichen Sozialversicherungsmodelle wenig zur Bedürfnisbefriedigung beitragen können. ${ }^{57}$

Auch für Lateinamerika ist typisch, daß die Landbevölkerung von Systemen sozialer Sicherheit kaum oder nur unzureichend erfaßt ist. ${ }^{58}$ Die spezifischen Probleme lassen sich stichwortartig wie folgt umschreiben: Die ökonomische Schwäche des Agrarsektors verhindert eine solide Finanzierung sozialer Sicherheit über Beiträge. Viele Arbeitgeber entziehen sich ihren Verpflichtungen, ihre Versicherten anzumelden und die entsprechenden Beiträge abzuführen. Dies ist einmal dadurch bedingt, daß bei vielen, vor allem den zahllosen kleinen und mittleren Arbeitgebern auf dem Lande die Voraussetzungen für die Erfüllung dieser Pflichten (z. B. Buchführung) fehlen, zum anderen steckt dahin-

55 Zu risk-pooling als wesentlichem Element von social security vgl. Rys, V., Comparative Studies of Social Security: Problems and Perspectives, in: Bulletin of the International Social Security Association, 1966, S. 249.

56 Vgl. International Social Security Association (Hg.), Social Security Protection of the Rural Population in Developing Countries, New Delhi 1980. Asociacion internacional de la seguridad social (Hg.), La protección social de la población rural, Buenos Aires 1981.

57 Mallet, A., Sozialschutz der Landbevölkerung, in: Internationale Revue für·soziale Sicherheit, 1980, S. $396 \mathrm{ff}$

58 Mallet, A., Situacion actual de la protección social de la población rural en America, in: AISS (Fn. 56), S. 10-21. 
ter häufig eine bewußte Verweigerung, weil die Arbeitgeber angesichts geringer Kontrollen damit rechnen können, daß ihre Nichterfüllung unbemerkt bleibt. Ein weiteres Problem liegt darin, daß die Entlohnung häufig vollständig oder zumindest teilweise in Naturalien erfolgt, so daß sich die Frage stellt, wie diese Naturalien im Hinblick auf die Beitragszahlungen bewertet werden sollen. Daß diejenigen Bevölkerungsteile, die noch in der Subsistenzwirtschaft tätig sind, ohnehin völlig ausscheiden, braucht der Vollständigkeit halber nur noch erwähnt zu werden.

Daraus wird ersichtlich, daß eine über Beiträge finanzierte und gar auf das Äquivalenzprinzip gestützte Reform sozialer Sicherheit für die Landbevölkerung eine besondere Härte darstellen muß. Es ist deshalb heute eigentlich fast einhellige Meinung, daß zum Schutze der Landbevölkerung andere Finanzierungsformen gefunden werden müssen. ${ }^{59}$ All diese vorstehend aufgezeigten Probleme gelten natürlich in vollem Umfang auch für Chile. $20 \%$ des Arbeitskräftepotentials (labour force) sind in Chile in der Landwirtschaft tätig, genauso viel wie in der Industrie. ${ }^{60}$ Es ist deshalb gerechtfertigt zu sagen, daß die neue chilenische Rentenversicherungsreform an den in der Landwirtschaft Tätigen vorbeigegangen ist (ausgenommen natürlich diejenigen, die in größeren landwirtschaftlichen Unternehmen, z. B. Plantagen, tätig sind). Vor allem enthält das neue Gesetz keine Investitionsverpflichtung zugunsten unterentwickelter Regionen, wie dies seit langem einem Vorschlag für Investitionen mit Mitteln sozialer Sicherheit entspricht. ${ }^{61}$ Die Verwaltungsgesellschaften haben nach dem neuen Gesetz vielmehr darauf zu achten, daß sie nur in äußerst produktive Bereiche investieren. Die Möglichkeit und Notwendigkeit, die Politik der sozialen Sicherheit mit der regionalen Wirtschafts- und Sozialpolitik zu verbinden, ${ }^{62}$ ist erst gar nicht ins Auge gefaßt worden.

59 Zum Ganzen sehr eindrucksvoll Grego, R. O., Organizacion y administracion de los programas de protección social de la población rural, in: AISS (Fn. 56), S. $88 \mathrm{f}$.

60 World Development Report 1981, S. 171.

61 Mesa-Lago (Fn. 16), S. 183.

62 Grego, R. O. (Fn. 56), S. 98. 


\section{The Privatization of State Pension Schemes in Chili}

\section{By Maximilian Fuchs}

The history of social security run by the state in Chile goes back to the 1920s. Ever since, a sophisticated system of social security provisions with a high rate of coverage has been developed.

As is well-known, the political and economic framework changed dramatically after the military coup in 1973 . The new political leadership began to do away with the traditional structures and institutions in politics and the economy. The dominant model and guidelines, which the new military junta followed, have been essentially formulated by members of the so-called "Chicago school", the doyen of which is the Nobel-Prize winner Milton Friedman.

In a nutshell, the leading principle was to create the conditions for the functioning of a free market economy, where state activities are reduced to a necessary minimum. Needless to say, these goals could not be attained without fundamental changes in the field of social security. Neo-classical criticism of the welfare state prompted high-ranking officials in the Chilean public administration to reorganize the social security system by abandoning the classical pattern of social security.

The most striking measure following the new directions was the new act on old age and invalidity insurance (Decreto Ley Nr. 3500, of 13. 11. 1980). The new legislation replaces the old public scheme by introducing a private insurance model administered by private companies. Employees and (on a voluntary basis) self-employed persons are free to choose to which insurance company contributions should be made. In this way one hopes to stimulate competition on the insurance market. As a principle benefits in the case of old age and invalidity (including benefits for survivors) are dependent on the contributions paid by the insured and the interest on these contributions.

The article analyses the new legislation and attempts to show its consequences and impact on the social development of the country.

\section{Guiana - A French Department like the Others?}

\section{By Frank Schwarzbeck}

French Guiana is today the only continental country in the Third World which has a considerable surface area and is still completely part of a European State. It is the largest of the remaining French overseas departments and overseas territories, which were formally integrated into metropolitan France after the Second World War. This paper presents Guiana's unusual history, the consequences of departmentalization, France's interests in this country, and its future prospects. 